\title{
Experiments with a Slow Neutron Velocity Spectrometer*
}

\author{
C. P. BAKer AND R. F. BACher
}

Cornell University, Ithaca, New York

(Received December 30, 1940)

\begin{abstract}
Neutron bursts were produced by modulation of the arc source of a small cyclotron. Neutrons subsequently slowed down in paraffin were detected by a $\mathrm{BF}_{3}$ ionization chamber and linear amplifier. Modulation of an additional amplifier allowed the counting of all detected.neutrons as well as those arriving in a specified time interval. Time of flight experiments were carried out at 3.0 meters with "on" times of 50 or $100 \mu \mathrm{sec}$. (microseconds) out of a period of $2500 \mu \mathrm{sec}$. The modulation of the source and detector was accurate to about $5 \mu \mathrm{sec}$. The absorption of thin $\mathrm{Cd}$ $\left(0.052 \mathrm{~g} / \mathrm{cm}^{2}\right)$ shows a resolved resonance absorption with maximum at $0.14 \mathrm{ev}$. The thick $\mathrm{Cd}\left(0.45 \mathrm{~g} / \mathrm{cm}^{2}\right)$ absorption was also examined and it was found that the absorption edge was not sharp, the transmission being 0.5 at 0.47 ev. Experiments with thick absorbers of $\mathrm{Rh}$ and In showed definite evidence of resonance absorption at 1.0
\end{abstract}

ev for both elements. Experiments were also carried out to study the delayed emission of thermal neutrons from the paraffin surrounding the source. An effective mean life of $170 \mu \mathrm{sec}$. was found. The effect of the resolution of the apparatus was examined and found to be small except for the resonances at $1.0 \mathrm{ev}$. The effect of the delayed emission of thermal neutrons on the time of flight measurements was also examined. From the thin $\mathrm{Cd}$ absorption curve $\Gamma$, the total width of the resonance level, was found to be $0.12 \mathrm{ev}$ and from the cross section at resonance

$$
[1 \pm(1 / 2 i+1)] \Gamma_{n},
$$

where $\Gamma_{n}$ is the neutron width and $i$ is the angular momentum of the absorbing nucleus, it was found to be $5 \times 10^{-4} \mathrm{ev}$. In this calculation 13 percent abundance of the effective isotope was assumed.
$I^{\top}$ $T$ is now well known that the absorption of slow neutrons by various nuclei changes rapidly with the kinetic energy of the neutron. All known nuclei show an absorption of slow neutrons which increases with decreasing energy and in addition many show the property of selective absorption for slow neutrons which are believed to lie in a narrow kinetic energy band. These absorption bands are believed to represent states of a compound nucleus and their width is believed to be roughly $10^{-8}$ of the excitation energy. Through the use of slow neutrons, one is permitted to get a tremendously magnified view of a small section of the energy spectrum in the region of $8 \times 10^{6} \mathrm{ev}$, the binding energy of a neutron. In principle it is possible to get a detailed picture of these absorption bands and thus to obtain useful information for the study of nuclear structure. There are, however, no monoenergetic sources of slow neutrons. Neutrons of the proper energy for study of these resonance effects are produced from the fast neutrons generated in nuclear disintegrations, by subjection of the neutrons to repeated energy losses. In this fashion a continuous neutron energy spectrum is produced and in order to investigate nuclear properties as a function of neutron

* This work was reported previously in brief. Baker and Bacher, Phys. Rev. 57, 351 (1940); 57, 1076 (1940). energy, the energy of neutrons for the particular process under investigation must be determined either by some direct method or by some indirect method.

A number of indirect methods of ascertaining the energy of slow neutrons have been developed, of which the most useful is probably the boron method which is founded on the very reasonable assumption that the absorption by boron is inversely proportional to the neutron velocity. The first direct method was the mechanical velocity selector of Dunning, Pegram, Fink, Mitchell and Segrè. ${ }^{1}$ Two sets of cadmiumcovered sector disks, separated by a given distance, absorbed neutrons in a given velocity interval which was determined by the speed of rotation of the disks. This selector was used in the neighborhood of thermal energy $(0.025 \mathrm{ev})$ and in order to obtain much higher energy neutrons, excessive rotational speeds would have been necessary. The resolution of the selector depends both upon the geometrical arrangements and upon the available neutron intensity which, with the radon sources used, was not very large.

With the introduction of large artificial sources of neutrons, direct control of the production of

${ }^{1}$ Dunning, Pegram, Fink, Mitchell and Segrè, Phys. Rev. 48, 704 (1935); Phys. Rev. 49, 103 (1936); Fink, Phys. Rev. 50, 738 (1936). 
neutrons was made possible. With such a modulated source, methods of measuring the neutron time of flight for a given distance were developed by Alvarez ${ }^{2}$ and by Fertel, Gibbs, Moon, Thomson, and Wynn-Williams ${ }^{3}$ (referred to as FGMTW). Alvarez used the $37^{\prime \prime}$ California cyclotron and produced the desired modulation by control of the plate voltage on the oscillator tubes. Neutrons were detected by a $\mathrm{BF}_{3}$ ionization chamber located at a distance and the amplifier output was short-circuited except during a certain interval after the burst. Modulation of the oscillator required large amounts of power and the bursts which were produced were of rather long duration and somewhat inaccurately defined, but the experiments which were carried out clearly demonstrated the power of the method. Alvarez found it possible to work with neutrons which had energies up to about $0.025 \mathrm{ev}$ although the resolution was rather low at this energy. FGMTW produced bursts of neutrons by modulating the ion source of a high voltage tube in which deuterons were accelerated and subsequently used to produce neutrons. While Alvarez counted only those neutrons which arrived during a certain interval after the burst, FGMTW obtained a continuous record of neutron arrival by use of an oscillograph. Primarily because of the fact that the modulation of the source was much easier to control, they were able to extend the time of flight measurements to energies of about $0.1 \mathrm{ev}$.

The present work is based on the use of the arc type ion source ${ }^{4}$ in the cyclotron and neutron bursts were produced by modulation of this source. The method of modulation therefore was in principle similar to that used by FGMTW. It seemed difficult, however, to get the desired accuracy in determination of the time of flight from an oscillograph record and hence a modulated amplifier in principle similar to that employed by Alvarez was used. A schematic diagram of the apparatus used in the present work is shown in Fig. 1. Neutrons produced by the cyclotron (1.4-Mev deuterons on $\mathrm{Be}$ ) were first slowed down in paraffin and then detected by a

\footnotetext{
${ }^{2}$ L. W. Alvarez, Phys. Rev. 54, 609 (1938).

${ }^{3}$ Fertel, Gibbs, Moon, Thomson and Wynn-Williams, Proc. Roy. Soc. 175, 316 (1940); Nature 142, 829 (1938).

${ }^{4}$ Livingston, Holloway and Baker, Rev. Sci. Inst. 10, 63 (1939).
}

$\mathrm{BF}_{3}$ ionization chamber connected to a linear amplifier which operated continuously. The input thyratron of the discriminating amplifier flashed for each neutron detected and the total number of flashes counted through the scale-of-16 counter. The pulses arriving in the desired time interval were selected by the discriminating amplifier which was controlled by the amplifier modulator. The design and operation of the various components of the apparatus will be discussed below.

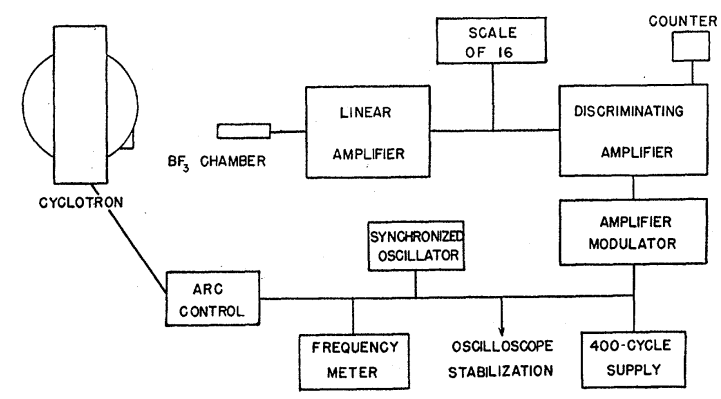

FIG. 1. Schematic diagram of apparatus.

\section{Source}

\section{Apparatus}

From previous work on the development of the arc type ion source it was known that this source would operate in the cyclotron from an alternating supply. Preliminary experiments on source modulation were therefore undertaken with the arc operating from a 400-cycle generator. In order to find out how well this operation controlled the deuteron beam, the target was grounded through a resistance of $10^{4}$ or $10^{5} \mathrm{ohms}$ and connected to an oscilloscope. With the oscilloscope sweep synchronized to the a.c. supply, square pulses which rose and fell in 10 microseconds or less, were observed and at this stage it was quite possible that the observed width of rise or fall was due to the amplifier which was used with the oscilloscope tube.

It is necessary to be able to regulate the duration of the deuteron burst. This was first done by connecting the arc in series with a thyratron used in a conventional grid-controlled rectifier circuit. The same alternating potential was applied to the grid and plate of this thyratron except that the phase of the potential applied to the former was shifted so that the grid was held negative over part of the operating cycle. The arc was thus 


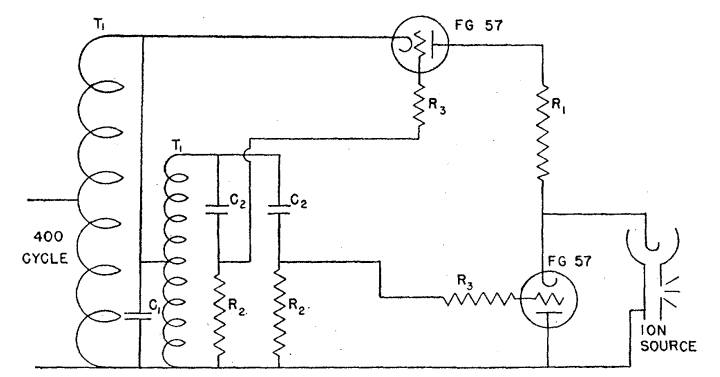

FIG. 2. Arc control circuit. $R_{1}=50$ adjustable; $R_{2}=30,000$ variable; $R_{3}=30,000 ; C_{1}=1 ; C_{2}=0.01$. Resistance in ohms, capacity in $\mu \mathrm{f}$.

started when the thyratron fired and extinguished when the applied potential became too low. This method has the disadvantage that the critical extinguishing potential and hence the time of extinguishing, depends upon the gas pressure and filament condition in the arc and these are not stable over long periods of time.

It appeared necessary both to start and stop the arc by some thyratron control. The circuit finally adopted is shown in Fig. 2. The upper thyratron starts the arc discharge at some time in the cycle determined by the phase of the applied grid potential and the lower thyratron shortcircuits the arc and thereby stops the discharge. The timing of these two events is controlled by the phase-shifting network $T_{1} R_{2} C_{2}$. Both thyratrons are extinguished when the supply voltage changes sign. The resistor $R_{1}$ is used to prevent excessive currents when both tubes are conducting. For most stable operation the grid potential should vary rapidly with time in the critical region so that changes in the supply voltage or the firing voltage of the thyratron will have little effect upon the time of firing. The large grid swing ( 400 volts) used to produce this rapid variation requires the use of the grid resistors $R_{3}$ in order to limit the grid current and prevent damage to the tubes. None of the circuit constants was critical except, of course, the phase shifting components which had to be adjusted for the desired duration of the burst. With the 400cycle modulation used throughout this work, the pulse applied to the arc could be varied from 50 microseconds $(\mu \mathrm{sec}$.) to somewhat more than $500 \mu \mathrm{sec}$. The voltage pulse controlling the arc rose or fell within $5 \mu \mathrm{sec}$.

The modulation of the deuteron beam controls the production of fast neutrons but delays may be introduced in the slowing down process. The slowing down took place in a paraffin block in which the Be target was imbedded as shown in Fig. 3. The detecting chamber for the time of flight measurements was placed on a line approximately perpendicular to the face of the paraffin block. Since the average energy of the neutrons is reduced to $E / e^{n}$ by $n$ collisions with the hydrogen in the paraffin, about 16 collisions are necessary to reduce the energy from $5 \mathrm{Mev}$ to $1 \mathrm{ev}$. The variation of the mean free path with neutron energy somewhat complicates the calculation of the time of slowing down. Assuming a mean free path of $5 \mathrm{~cm}$ for $E>5000 \mathrm{ev}$ and $1 \mathrm{~cm}$ for $5000 \mathrm{ev}>E>0.1 \mathrm{ev}$, it takes $0.13 \mu \mathrm{sec}$. for a neutron to go from $5 \mathrm{Mev}$ to $5000 \mathrm{ev}, 1.5 \mu \mathrm{sec}$. to go to $1.4 \mathrm{ev}$ and $4.0 \mu \mathrm{sec}$. to go from $5 \mathrm{Mev}$ to $0.19 \mathrm{ev}$. In general the slowing down time will be of the order of the time for a few mean free paths at the final velocity or a few $\mathrm{cm}$ and this distance is small compared to the distance of source to detector.

Somewhere in the region of $0.25 \mathrm{ev}$, the assumptions made above break down because the energy loss per collision is affected by the presence of the hydrogen chemical bond. In addition, the neutrons at still lower energy are in thermal equilibrium with the paraffin and the delay in emission will depend upon their effective mean life in the paraffin. This latter point was investigated and will be discussed later.

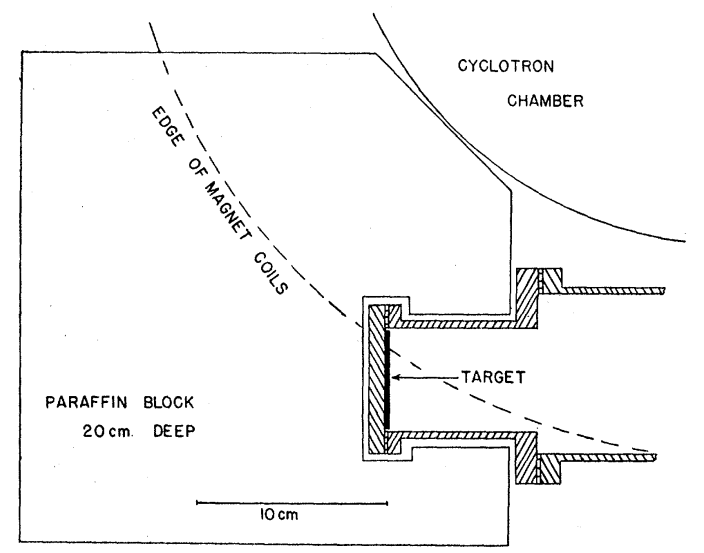

FIg. 3. Target arrangement. Observations were made on a line perpendicular to the face of the paraffin indicated at the bottom of figure. 


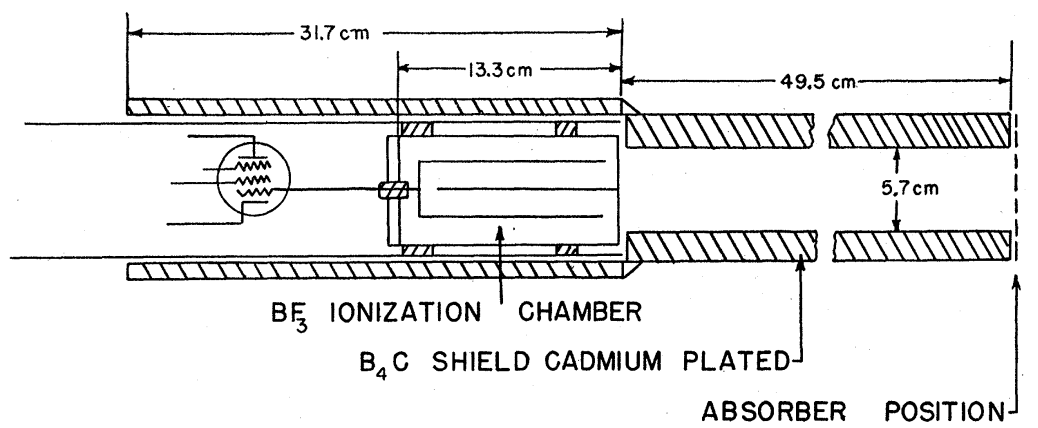

FIG. 4. Ionization chamber and boron carbide collimator.

\section{Detector}

The slow neutrons were detected with a $\mathrm{BF}_{3}$ ionization chamber and linear pulse amplifier. The ionization chamber was cylindrical with inside diameter of $5 \mathrm{~cm}$ and $15 \mathrm{~cm}$ long and was filled with $\mathrm{BF}_{3}$ gas at atmospheric pressure. A collecting potential of 800 volts was used to collect negative charges. The chamber was shielded with cadmium and boron carbide and has a collimating tube as shown in Fig. 4 . The boron carbide of the collimating tube was about $1 \mathrm{~g} / \mathrm{cm}^{2}$ and about half as much for the chamber shield. Because of the geometrical arrangement and the presence of the first amplifier tube, there was no boron carbide shielding at the back of the ionization chamber although there was a cadmium shield not indicated in Fig. 4. The extension of the boron carbide shield then allowed cadmium penetrating neutrons to come from only a small solid angle. The general position of the ionization chamber with respect to the walls of the room and the cyclotron is shown in Fig. 5. The ionization chamber was placed so that in back it could see only an adjacent room, connection to the amplifier being made by a $1.5-\mathrm{m}$ flexible cable. The collimation in front was such that at $3 \mathrm{~m}$ from the source, the view of the ionization chamber was restricted almost entirely to the face of the paraffin block shown in Fig. 3 .

Certain special precautions must be observed in the use of the ionization chamber and linear amplifier in time of flight measurements if the time of recording a neutron pulse at the output of the amplifier is to be an accurate measure of the time of arrival of the neutron in the ionization chamber. The collection of negative ions in a $\mathrm{BF}_{3}$ chamber had been found to give rise to much sharper pulses, as might be expected if the negative ions are electrons. This collection time for positive ions is about $250 \mu$ sec. and for electrons about $6 \mu \mathrm{sec}$. Since the collection time is dependent on the position of the disintegration in the chamber, it is highly desirable to make this time as short as possible.

Certain modifications were made in the linear amplifier in order to obtain speed of response and avoid excessive smearing of the sharp pulses from the ionization chamber. To do this it is necessary to increase the frequency band passed by the amplifier. The time constants of the interstage coupling elements were reduced by decreasing the size of the coupling condensers to $0.001 \mu \mathrm{f}$. This did not give sufficient high frequency response, so it was necessary to reduce the plate and grid resistors to 50,000 ohms. In order not to reduce the gain per stage, tubes with a high amplification factor were substituted for the conventional type 6C6. These tubes were type 1851, with an amplification factor of 6000 , and were used in the second and third stages. The shielded wire in the cable between the first and second tubes had a capacity of $3 \mu \mu \mathrm{f}$ per $\mathrm{cm}$, and it was found necessary to substitute coaxial cable which had a capacity of a tenth this amount. The wider response of the amplifier made the signal to noise ratio somewhat less than that in conventional amplifiers. This is unavoidable as the noise is roughly proportional to the band width of the amplifier.

Even with these steps taken to increase the amplifier speed a definite time lag was found. A sketch of a neutron pulse as it appears on an oscilloscope screen is shown in Fig. 6. While the pulse seems to start at a time $T_{1}$, it is not re- 
corded until $T_{2}$ when the potential rises to the flash point of the thyratron. The time lag $T_{2}-T_{1}$, was estimated to be about $25 \mu$ sec. In the course of the experiments it was found possible to make a measurement of the over-all time lag and this is considered to be more accurate.

The output of the linear amplifier was fed into a separate unit called the discriminating amplifier which is shown in Fig. 7. The input thyratron (type 885) flashed for all neutrons detected. The bias of this tube was adjusted in the usual way to eliminate the noise background and was not changed in the course of an experiment. The thyratron output was fed into two channels. The first of these was connected to a scale-of-16 counter and recorded all neutrons detected. The total number of neutrons detected was used as a measure of the integrated beam intensity and has several advantages over the usual method of beam current integration. Changes in the sensitivity of the detector or efficiency of the target do not, to a first approximation, affect the results if the number of timed neutrons is always referred to the total number. The 56 tube in this channel was used principally as a buffer tube to isolate the output circuit of the thyratron from possible interaction with the scale counter or external disturbances. The second channel was the timing channel, and led to the grid of the type 57 tube. The bias on this tube was adjusted so that the amplification was a critical function of the screen potential. The screen potential was supplied by a square wave generator (amplifier modulator) whose output was adjustable in width and phase so that only those pulses which occurred in a predetermined time interval were amplified. The following 56 was used to amplify and invert the

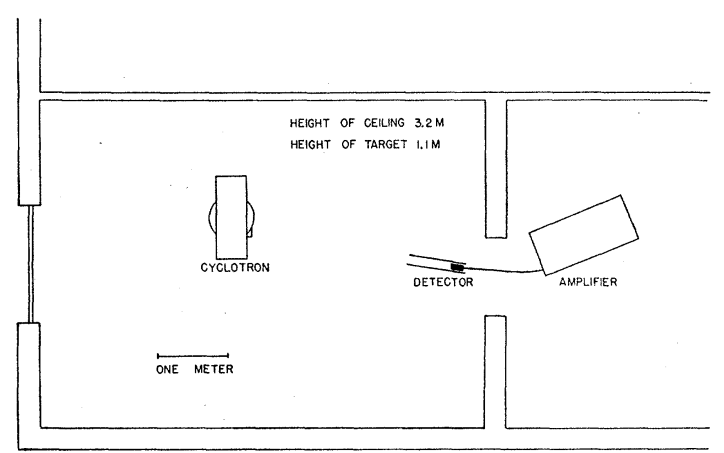

FIG. 5. Room plan. timed pulses and the final 885 operated the mechanical counter which recorded the number of timed neutrons. The circuit components of the output of the first 885 were chosen so that the pulses into the discriminating tube were sharp and of uniform height, which simplified the modulation problem.

The square wave generator or amplifier modulator is shown in Fig. 8. The firing of the first 885 starts the pulse which controls the discrimination and the firing of the second tube stops it by short-circuiting, in a similar manner to the arc control. The time of starting and stopping is determined by the phase-shifting network $T_{1} C_{2} R_{4}$ and since the control of each tube is roughly independent of the other, any desired phase and width of pulse may be obtained. The power supply indicated as a battery must float, but a transformer rectifier system was finally used. The time taken for the pulse to rise from zero to its full value, or to fall, is less than 5 microseconds. The same considerations involving stability that apply to the arc control apply here, so the grid swing used was large (300 to 500 volts).

\section{Calibration}

All time measurements were made on the screen of a 5-inch oscilloscope which was synchro-

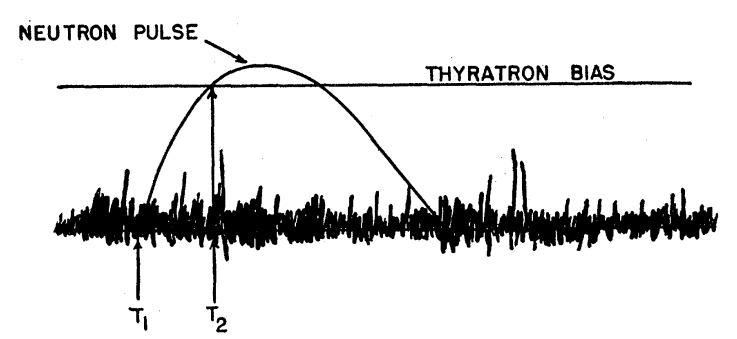

FIG. 6. Sketch of oscilloscope trace showing neutron pulse which starts at $T_{1}$ and fires the thyratron at $T_{2}$.

nized to the 400-cycle supply. For accurate measurements the oscilloscope sweep could be expanded in any region. With a 5-inch sweep the time scale was normally $500 \mu \mathrm{sec}$./inch and this was frequently increased to $100 \mu \mathrm{sec}$./inch. A central tap switch permitted one to examine the arc control, amplifier modulator, oscillator or various outputs with the oscilloscope. Time settings with the arc control and amplifier modulator could be made to better than 0.05 


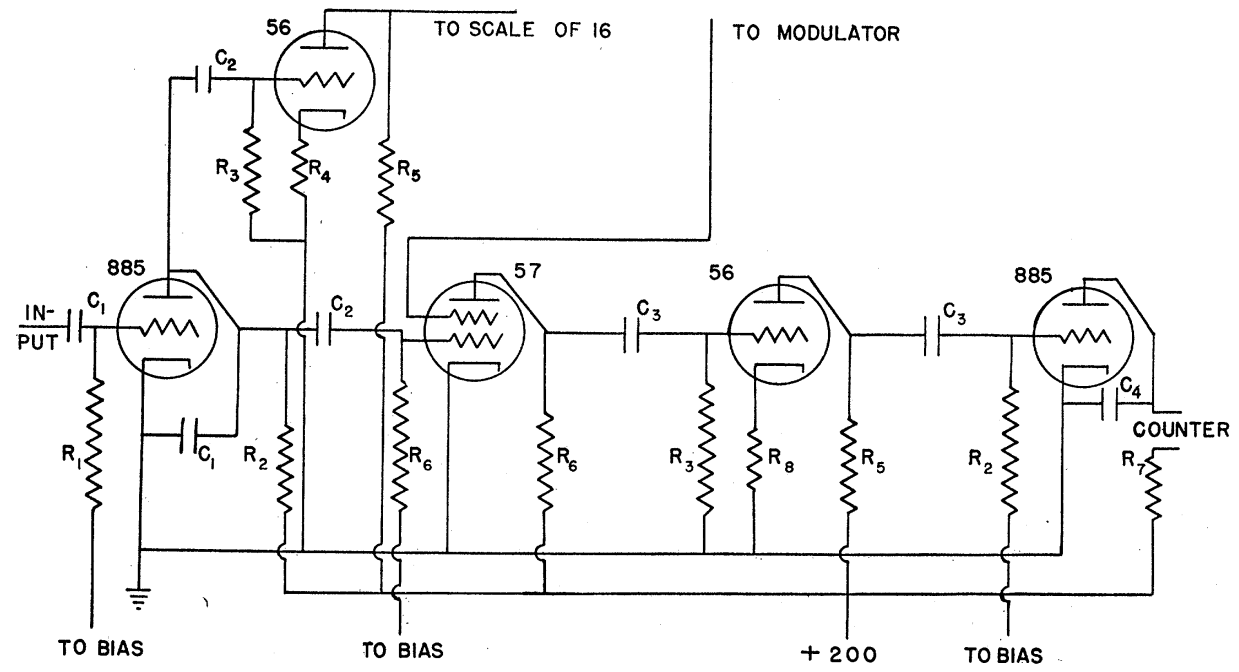

FIG. 7. Discriminating amplifier circuit. $R_{1}=40,000 ; R_{2}=200,000 ; R_{3}=100,000 ; R_{4}=4000$; $R_{5}=30,000 ; R_{6}=20,000 ; R_{7}=10,000$ adjustable; $C_{1}=0.00025 ; C_{2}=0.0001 ; C_{3}=0.005 ; C_{4}=1$. Resistance in ohms, capacity in $\mu \mathrm{f}$.

inch on the oscilloscope screen which corresponded to $5 \mu \mathrm{sec}$.

Since a strictly linear sweep is difficult to obtain and the sweep frequently used in an expanded, nonlinear condition, all time measurements were referred to a 10-kilocycle oscillator synchronized to the 400-cycle supply. This was used to calibrate the oscilloscope trace. For greater accuracy, time measurements were made in $50-\mu$ sec. units, corresponding to one loop on the oscillator wave ; i.e., the source time, detector time and interval between were set to be a certain number of $50-\mu \mathrm{sec}$. loops. The oscillator used was the so-called "transitron" ${ }_{5}$ and the synchronization was introduced on the control grid of the 57 tube normally connected to the cathode.

The frequency of the 400-cycle motor generator set was checked against a separate stable oscillator of known frequency. It was found that the generator frequency was constant to about 1 percent.

It is important to know the time lag of the entire time measuring apparatus. In addition to the time lag in the amplifier, there is a lag of the order of $10 \mu \mathrm{sec}$. due to the acceleration of the deuterons. This lag depends on the cyclotron " $D$ " potential, the $10-\mu$ sec. estimate being based on the assumption of 100 revolutions of ions at a

\footnotetext{
${ }^{5}$ C. Brunetti, Rev. Sci. Inst. 10, 85 (1939).
}

frequency of 10 megacycles. The sum of all the time lags was measured directly by finding the time interval between production of the arc pulse and the detection of the first neutrons at the detector. With no time lags, this interval should be approximately zero for the very fast neutrons which first reach the detector in the same position used for time of flight measurements. In order to increase the number of fast neutrons the paraffin was removed from around the $\mathrm{Be}$ target. The detector time was then adjusted to be just before the source time and the detector time changed until it overlapped the source time. Counts were taken as a function of the detector time and the results are shown in Fig. 9. With $30 \mu \mathrm{sec}$. overlap of the source and detector the observed count was apparently just background, but for greater overlap the number

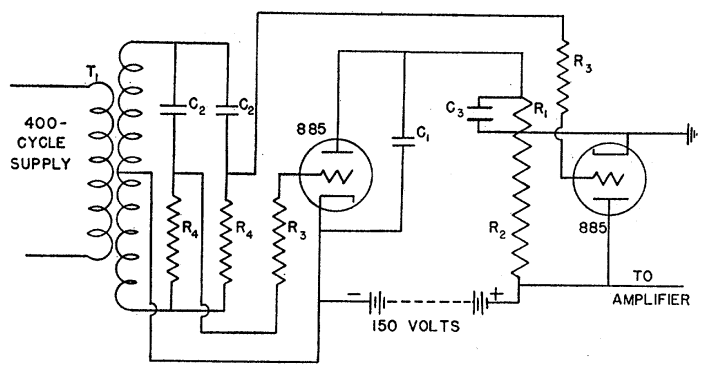

FIG. 8. Amplifier modulator circuit. $R_{1}=10,000$; $R_{2}=50,000 ; R_{3}=500,000 ; R_{4}=20,000 ; C_{1}=0.1 ; C_{2}=0.02$; $C_{3}=0.005$. Resistance in ohms, capacity in $\mu \mathrm{f}$. 


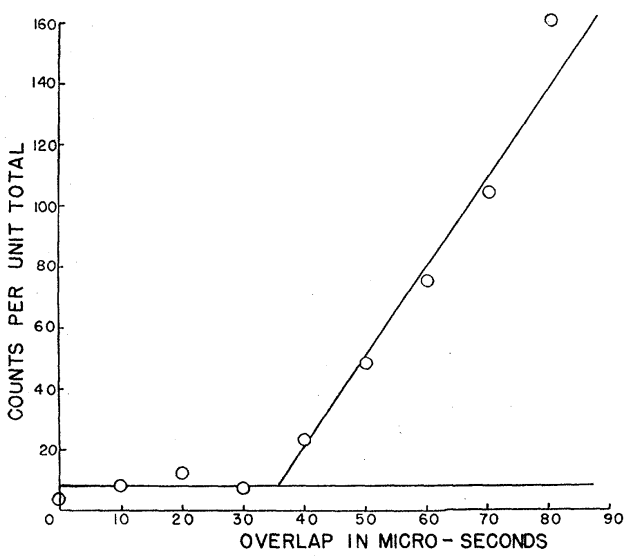

FIG. 9. Determination of over-all time lag. Neutrons counted vs. overlap of "on" times of source and detector.

of counts increased approximately linearly. The linear increase was extrapolated to the background and an effective time lag of $35 \mu \mathrm{sec}$. obtained. This time lag must be subtracted from all time measurements. It is gratifying but possibly fortuitous that this figure agrees with the sum of the amplifier lag and estimated lag in the cyclotron.

\section{Experimental Procedure}

The apparatus was put into operation at first with the cyclotron ion source unmodulated and the amplifier was then adjusted for the best ratio of counting rate of neutrons to background. The ion source was next operated from the arc control which was adjusted for the length of burst desired. When the ionization chamber was placed at the proper distance and the amplifier connected to the oscilloscope the neutron velocity spectrum could be seen, the group at thermal energies being particularly striking. The square topped wave which modulated the amplifier was then adjusted for the desired time of flight. Counting was started with a three-pole switch which simultaneously started the scale-of-16 counter, the timed counter, and an electric clock. The observations were timed in order to be able to correct for noise and contamination background. Counts were taken until the desired number of timed neutrons were recorded, the number being determined by the statistical accuracy desired and convenient to obtain, usually 10 percent. For convenience in recording, observations were made for an integral number of revolutions of the mechanical counter dial at the output of the scale-of-16 counter, or in units of 960 recorded neutrons. The number of timed neutrons was referred to the total number of neutrons recorded in the same time interval. The time settings were made by switching the controlling pulse and the synchronized oscillator alternately onto the oscilloscope screen so that a direct comparison was made. At the end of an observation the time settings were checked before a new adjustment was made, although it seldom happened that any unexpected shift occurred. For absorption experiments observations were made with and without the absorber in place, with no change in the timing so that if the settings were stable any error in the initial adjustment of the width of the setting had little effect on the measured absorption.

For absolute absorption measurements it was necessary to compare the untimed neutrons with and without the absorber. This was necessary because the timed neutrons were referred to the untimed and the latter represented different numbers of incident neutrons for the runs with and without absorber. In order to avoid errors due to change in intensity of the source, this comparison was made by a succession of 30second runs with and without absorber over a period of 10 minutes with the cyclotron operating under steady conditions.

It was found that with a thick boron carbide absorber $\left(2 \mathrm{~g} / \mathrm{cm}^{2}\right)$ in front of the ionization chamber, there was an appreciable background which decreased rapidly (Fig. 10), being approximately 5 percent at $300 \mu \mathrm{sec}$. after the end of the burst. This background is probably due to neutrons which are scattered around the room and which might penetrate the shielding and possibly be absorbed in the chamber. Such neutrons would, of course, take much longer to lose energy because of the long distance between collisions. The data were always corrected for this background.

Since it is known that the neutrons have a considerable life in the paraffin as thermal neutrons it is to be expected that thermal neutrons will be emitted after the neutron burst. This delayed emission has a considerable effect in these experiments and was therefore measured 
directly with the ionization chamber in contact with the paraffin. The results and their effect upon the resolution of the apparatus will be discussed. With the ionization chamber in the same position, the neutrons from the block were also investigated with a thick $\mathrm{Cd}$ absorber $(0.45$ $\left.\mathrm{g} / \mathrm{cm}^{2}\right)$ interposed. It was found that very few neutrons were detected after the production had stopped. If the gas pressure in the cyclotron was deliberately raised some neutrons were detected after the arc had stopped. With increasing gas pressure the number of these neutrons increased and the time after the extinguishing of the arc during which they were seen, increased until finally little effect of the arc modulation could be detected. When the gas pressure was too high a bright glow could be seen between the " $D$ 's" indicating that the unmodulated neutrons came from ions originating outside the arc. In these experiments, care was taken to operate the cyclotron chamber at sufficiently low pressure to avoid this difficulty.

\section{Results}

The data were first corrected for the noise and contamination background taking into account the fact that for the timed neutrons the amplifier was sensitive for only a small fraction of the time. The timed neutron counts were then corrected for the fast neutron background as shown in Fig. 10. In the experiments carried out at 3 meters, this introduced an appreciable correction only for those neutrons with energy above 0.5 ev. For the data without the absorber the number of fast neutrons per unit total was subtracted from the number of counts per unit total to obtain the final result. The data with absorber, after being corrected for noise, were multiplied by the ratio of the untimed totals so that the numbers correspond to the same number of incident neutrons from the cyclotron. The number of fast neutrons per unit total already computed was then subtracted directly to obtain the corrected number with absorber. The ratio of the corrected numbers with and without absorber then gives the transmission directly and with the known number of atoms per $\mathrm{cm}^{2}$ in the absorber, the cross section is obtained. The above procedure for the fast neutron background assumes that this background is the same with and with- out the absorber. Since it was rather laborious to do, this was not checked experimentally, but it seems very reasonable.

The time of flight associated with each point is the interval between the middle of the "on" time of the arc and the "on" time of the amplifier. These times were measured from the position of the controlling pulses on the oscilloscope screen and the $35-\mu$ sec. time lag was subtracted.

The results for a thin Cd absorber $(0.052 \mathrm{~g} / \mathrm{cm})$ are shown in Fig. 11. The points indicated as circles were taken with a source-detector distance of 3.0 meters and an "on" time of source and detector of $100 \mu \mathrm{sec}$. The points indicated by crosses were taken with a source-detector distance of 1.5 meters and a source and detector "on" time of $50 \mu \mathrm{sec}$. so the resolution is the same for both sets. The times of flight have in each case been divided by the distance of flight so the axis represents the time of flight for one meter. The absorption maximum has been clearly resolved, the maximum cross section appearing for a one-meter time of flight of $190 \mu \mathrm{sec}$. or an energy of $0.14 \mathrm{ev}$. There is a definite indication of an ill-defined minimum in the neighborhood of $0.03 \mathrm{ev}$ and below this energy the cross section seems to rise slowly. The statistical accuracy of each point is about 10 percent in the measurement of the transmission. No correction has been applied for the possible effect of the resolution of the apparatus or the effect of delayed emission of thermal neutrons, both of which are discussed later.

The absorption of thick cadmium $\left(0.45 \mathrm{~g} / \mathrm{cm}^{2}\right)$ was initially undertaken in order to get some experience at higher energy (see Fig. 12). Knowledge of the absorption is also useful because such a thickness of cadmium is often used

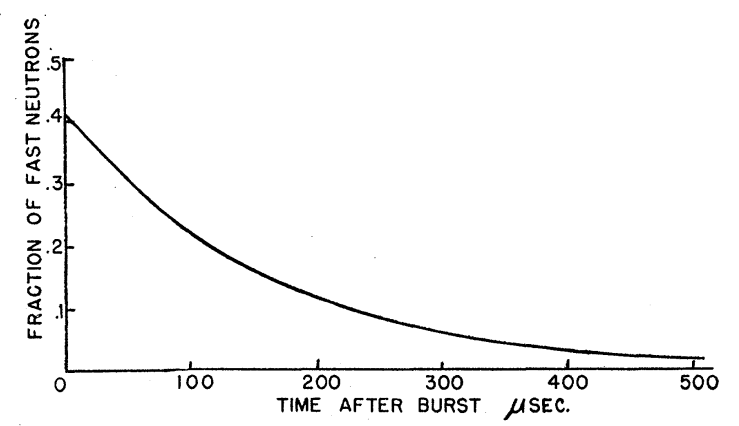

FIG. 10. Fast neutron background. 


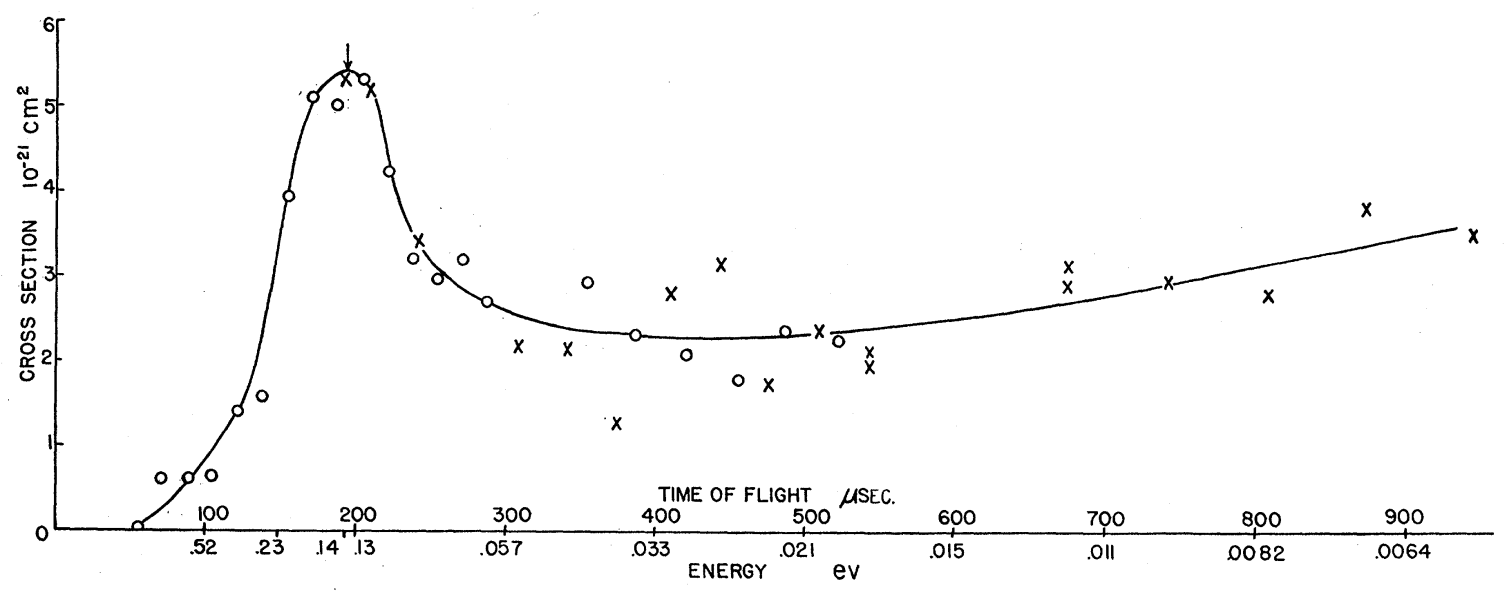

Fig. 11. Absorption of thin cadmium $\left(0.052 \mathrm{~g} / \mathrm{cm}^{2}\right)$. Cross section for all cadmium isotopes vs. time of flight for 1 meter with corresponding energy points shown below. $O: 3$ meters source detector distance; $X: 1.5$ meters.

as a screen in slow neutron experiments. These data were taken at a distance of 3.0 meters between source and detector and the "on" times were $100 \mu \mathrm{sec}$. The absorption at low energy is practically complete and extends to much higher energy than the thin cadmium absorption, being 0.5 at $0.47 \mathrm{ev}$. The absorption edge is not sharply defined and the effect of the resolution of the apparatus on this edge is not large as will be shown later. The statistical accuracy above $0.4 \mathrm{ev}$ is 10 percent but at lower energy it was decreased because of the long time involved in taking accurate data where the transmission is low. At 0.21 ev the error is 15 percent and the lowest energy point has a statistical error of 25 percent. No correction for delayed emission of thermal neutrons need be considered for these data as the energy range is above that where the thermal distribution plays an important role.

The results of the measurements of the transmission of indium and rhodium are shown in Figs. 13 and 14. These data were taken at a distance of 3 meters between source and detector and with source and detector "on" times of $50 \mu \mathrm{sec}$. which gave the highest resolution used in this work. Since these measurements were of an exploratory nature, thick absorbers $\left(0.39 \mathrm{~g} / \mathrm{cm}^{2}\right.$ for In and $0.60 \mathrm{~g} / \mathrm{cm}^{2}$ for $\mathrm{Rh}$ ) were used. The transmission curves clearly show the resolution of the resonances which are at approximately 1.0 ev for each absorber. The probable errors of about 10 percent for In and from 10 to 20 percent for $\mathrm{Rh}$, were governed largely by the long time it took to get data at high energy. For a single point on the Rh curve at $1.0 \mathrm{ev}$, counts were taken for about 40 minutes without interruption. It will appear that for high energy, a considerable correction for the resolution of the apparatus is necessary if a reasonable determination of the cross section as a function of energy is wanted. The present data are not sufficiently accurate to warrant these corrections and no attempt was made to make the measurements absolute.

The effective mean life or the delayed emission of thermal neutrons from the paraffin block was measured by placing the face of the ionization directly against the paraffin block. A burst of deuterons was produced for $50 \mu \mathrm{sec}$. and neutrons were counted in $50-\mu \mathrm{sec}$. intervals starting $15 \mu \mathrm{sec}$. after production had stopped. The results of this experiment are shown in Fig. 15 where the timed neutrons per unit total are plotted on a logarithmic scale against the time after the end of the burst. Except for the first two points, they seem to lie on a straight line indicating an exponential decay and an effective mean life of $170 \mu \mathrm{sec}$. The statistical accuracy of the data is better than 5 percent but in this measurement any error in adjusting the width of the detector "on" time enters linearly into the results. This error was not greater than $5 \mu \mathrm{sec}$. or 10 percent but it is the largest source of error in the results. 


\section{Resolution of Apparatus}

It is quite clear that experiments with higher resolving power would have given greater detail in the results, but this was not feasible with the present equipment largely because of intensity. In the absence of such information, it is desirable to examine in some detail how the results are affected by the resolving power of the apparatus. No entirely satisfactory method of correcting for the resolving power of the apparatus has been found, although several methods have been used to attack the problem which give interesting results and are of help in the evaluation of the data.

If there is a uniform production of neutrons for a time $\delta$ and recording of neutrons for the same interval at a time $s$ after the production of neutrons has stopped, neutrons having times of flight between $s$ and $s+2 \delta$ will be recorded and the most favored time of flight will be $s+\delta$. The function $f(s, t)$ expressing the distribution in time of flight to which the apparatus is sensitive starts from zero at $t=s$ and increases linearly until $t=s+\delta$ and decreases linearly until $t=s+2 \delta$. With the proportionality constant $C, f(s, t)$ is expressed by the following relations.

$$
f(s, t)= \begin{cases}0, & t<s, \\ (t-s), & s<t<(s+\delta), \\ 2 \delta-(t-s), & (s+\delta)<t<(s+2 \delta), \\ 0, & (s+2 \delta)<t .\end{cases}
$$

The distribution function $f(s, t)$ and its relation to the "on" times is shown in Fig. 16.

Fundamentally the problem of finding the

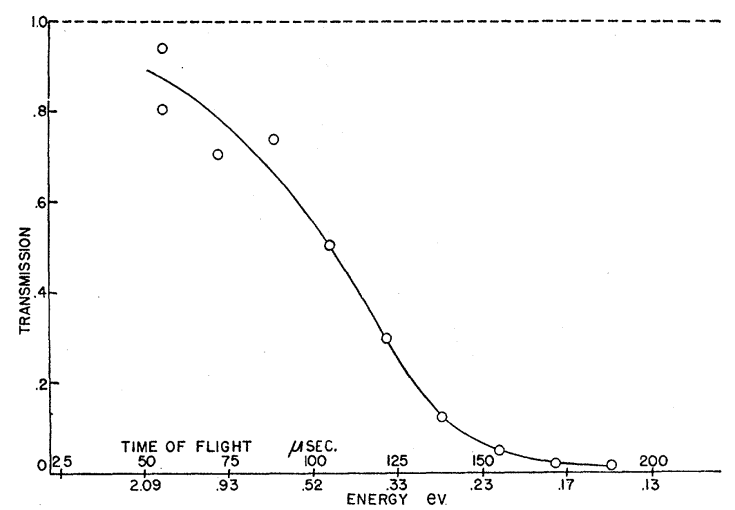

FIG. 12. Transmission of thick cadmium $\left(0.45 \mathrm{~g} / \mathrm{cm}^{2}\right)$ vs time of flight for 1 meter. Data were taken at 3 meters.

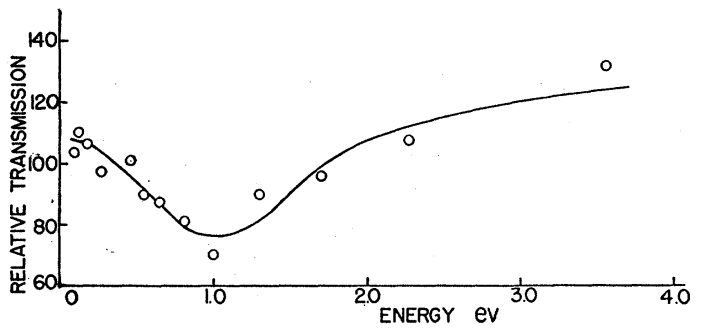

FIG. 13. Relative transmission of indium $\left(0.39 \mathrm{~g} / \mathrm{cm}^{2}\right) v s$. energy showing effect of the resonance at $1.0 \mathrm{ev}$. Data were taken at 3 meters.

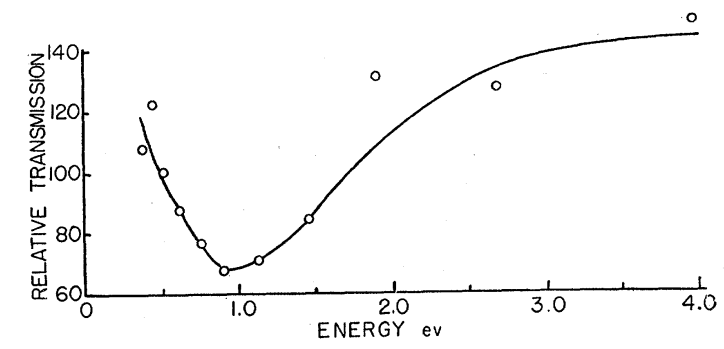

FIG. 14. Relative transmission of rhodium $\left(0.60 \mathrm{~g} / \mathrm{cm}^{2}\right)$ $v s$. energy showing effect of resonance at $1.0 \mathrm{ev}$. Data were taken at 3.2 meters.

actual number of neutrons, as a function of time of flight, from the observed data $D(s)$ amounts to finding a solution to the integral equation

$$
D(s)=C \int_{s}^{s+2 \delta} N(t) f(s, t) K(t) d t,
$$

where $f$ is the function just described, $N(t)$ is the neutron distribution from the source which is the function that is to be found, and $K(t)$ represents the variation in the efficiency of the detector with neutron energy. If $K(t)$ is known (for instance, it would be a constant times $t$ if the $1 / v$ law holds for boron) the analysis can be simplified by considering the product $K(t) N(t)$ as a single function $M(t) . f(s, t)$ can be considered as a function of $s-t$ which is zero outside the limits $s$ to $s+2 \delta$ so the integral equation can be written

$$
D(s)=C \int_{-\infty}^{\infty} M(t) f(s-t) d t .
$$

With the introduction of the Fourier transforms this equation can be solved for $M(t)$. This form of the solution is not, however, easily adapted to the practical problem since a function cannot easily be found to fit the data and in any case the labor involved is considerable and the data do not warrant such treatment. 


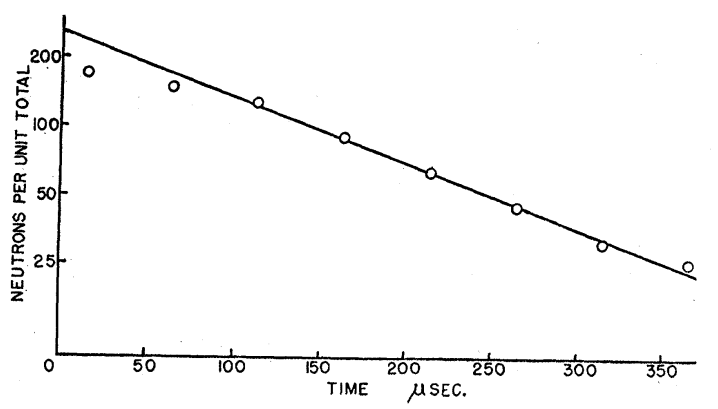

FIG. 15. Neutron decay in paraffin. Effective mean life $170 \mu \mathrm{sec}$.

The problem can be simplified by introducing an approximation for the function $M(t)$ in the form of the first three terms of the Taylor's expansion of $M(t)$ about $t_{0}$. Making a change of variable $t^{\prime}=t-(s+\delta)$, one has for the distribution function,

$$
\begin{aligned}
& f=0 \quad \text { for } \quad\left|t^{\prime}\right|>\delta \text {, } \\
& f=t^{\prime}+\delta \text { for }-\delta<t^{\prime}<0 \text {, } \\
& f=\delta-t^{\prime} \text { for } 0<t^{\prime}<\delta \text {. }
\end{aligned}
$$

Without the primes, $D$ becomes

$$
\begin{aligned}
D\left(t_{0}\right)=C \int_{-\delta}^{\delta} f(t)\left[M\left(t_{0}\right)\right. & +t M^{\prime}\left(t_{0}\right) \\
& \left.+\frac{t^{2}}{2} M^{\prime \prime}\left(t_{0}\right)+\cdots\right] d t .
\end{aligned}
$$

The value of the constant $C$ is not important as it is fixed for any $f$, and the transmission which is the interesting datum is the ratio of two observations of $D$. $C$ can be evaluated by considering $K(t)$ and $N(t)$ to be constant and unity, the observation then representing a unit number of neutrons.

$$
1=C \int_{s}^{s+2 \delta} f(s, t) d t=C \delta^{2} \quad \text { or } \quad C=1 / \delta^{2} .
$$

The integration gives

$$
D=M \delta^{2}+M^{\prime \prime} \delta^{4} / 12+\cdots .
$$

The absence of a term in $M^{\prime}$ in this expression is due directly to the symmetry of $f(s, t)$ about $t=t_{0}$. In obtaining a transmission curve the ratios of the numbers with and without the absorber are considered, so the interesting information is $T=D_{2} / D_{1}$. The analysis is the same for the two cases except that for $D_{2}$ the corresponding func- tion $M_{2}$ is not the number of neutrons from the source but is the number transmitted by the absorber or $M_{2}=A(t) M_{1}$ where $A$ is the transmission of the absorber. The measured transmission is then expressed by

$$
T=\frac{\begin{array}{c}
A(t) M(t) \delta^{2}+\left(\delta^{4} / 12\right)\left[A^{\prime \prime}(t)\right. \\
\left.+2 M^{\prime}(t) A^{\prime}(t)+A(t) M^{\prime \prime}(t)\right]
\end{array}}{M(t) \delta^{2}+\left(\delta^{4} / 12\right) M^{\prime \prime}(t)} .
$$

In powers of $\delta$, this may be written

$$
\begin{array}{r}
T=A+\left[\left(A^{\prime \prime}+2 A^{\prime} M^{\prime}\right) / M\right]\left(\delta^{2} / 12\right) \\
+ \text { higher powers. }
\end{array}
$$

If the resolution width is small the true transmission is given directly by the data as might be expected. To a second approximation the data are unaffected by the slope of the curve $A$, if the function $M$ is large and slowly varying with energy. $M$ is the product of the number of neutrons from the source and the efficiency of the detector and is therefore the effective number of neutrons or the number seen by the detector. Under these conditions only the curvature of the transmission curve will alter the data. In the vicinity of a resonance line the curvature may possibly be so great that the data do not give a good picture of the true transmission unless the resolution of the experiment is sufficiently increased.

In order to get a clearer picture of the effect of resolution on the data, the problem was approached from the opposite direction. Instead of attempting a solution of Eq. (1) for $N(t)$ in terms of the data, hypothetical curves for $N(t)$ were plotted and the corresponding functions $D(s)$ were computed for several values of $\delta$ which determines the resolving power. The BreitWigner theory predicts that the cross section in

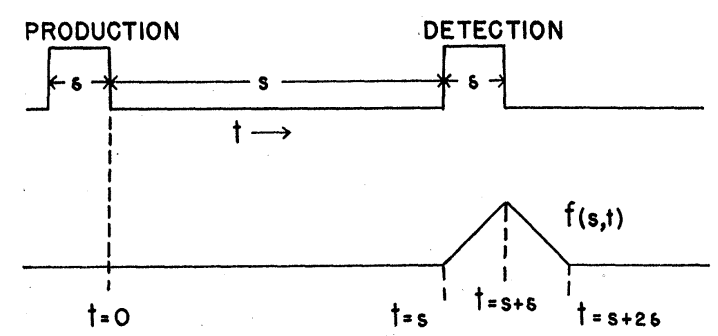

FIG. 16. Distribution function $f(s, t)$ showing relation to "on" times of source and detector. 
the neighborhood of a resonance absorption is given in terms of the resonance energy $E_{R}$ and the total width $\Gamma$ by the following expression ${ }^{6}$

$$
\sigma(E)=\sigma_{0}\left(E_{R} / E\right)^{\frac{1}{2}} \Gamma^{2} /\left[\Gamma^{2}+4\left(E-E_{R}\right)^{2}\right]
$$

where $\sigma_{0}$ is the cross section at $E=E_{R}$.

$$
\sigma_{0}=\frac{\pi}{2}\left(1 \pm \frac{1}{2 i+1}\right) \frac{\hbar^{2}}{2 M E_{R}} \frac{4 \Gamma_{N}}{\Gamma} \text {. }
$$

$\Gamma_{N}$ is the neutron width and $i$ the angular momentum of the absorbing nucleus. The transmission of an absorber which has such a cross section will be

$$
T(E)=e^{-\sigma n},
$$

where $n$ is the number of atoms per $\mathrm{cm}^{2} . T(E)$ was then plotted on an axis representing time of flight for 1 meter. The value of $\sigma n$ was chosen such that there was almost complete absorption at resonance, since thick absorbers were actually used for indium and rhodium. The energy $E_{R}=1.0 \mathrm{ev}$ was also picked to correspond to this case. Curve 0 in Figs. 17 and 18 is for $T(E)$ under these circumstances with $\Gamma=0.2$ and $\Gamma=0.4 \mathrm{ev}$, respectively. Curves 1 and 2 represent the transmission which would be observed for the resolution indicated by the distribution functions shown at the bottom of each plot. They were computed by approximate numerical evaluation of the integral in Eq. (1) for a number of values of $s$ and a smooth curve drawn through the points. These curves correspond to "on" times

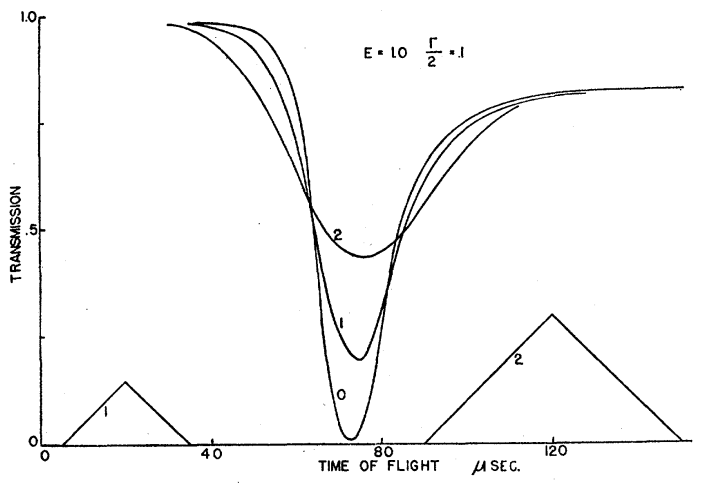

FIG. 17. Dispersion curve for $\Gamma=0.2$ and minimum transmission at $1.0 \mathrm{ev}$ (curve 0 ). Effect of resolution by distribution functions indicated, is shown in curves 1 and 2 .

${ }^{6}$ H. A. Bethe, Rev. Mod. Phys. 9, 69 (1937). See Eq. (428).

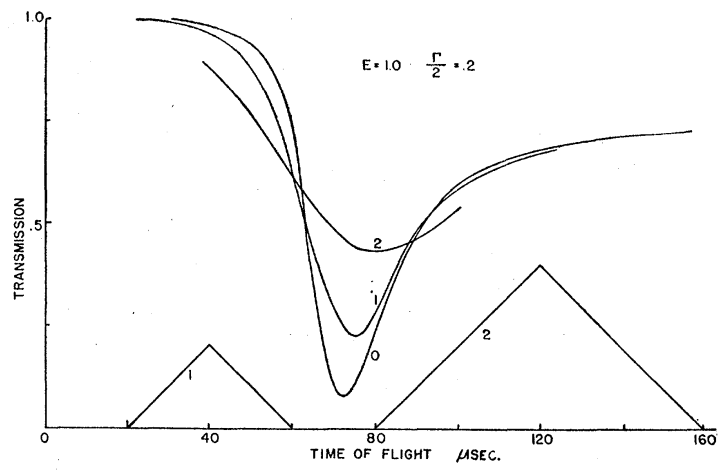

FIG. 18. Dispersion curve for $\Gamma=0.4$ and minimum transmission at $1.0 \mathrm{ev}$ (curve 0 ). Effect of resolution by distribution functions indicated, is shown in curves 1 and 2 .

for 3 meters of 45 and $90 \mu$ sec. for Fig. 17 and of 60 and $120 \mu$ sec. for Fig. 18. Curve 1 in each case corresponds approximately to the resolution which was actually used for indium and rhodium. The effect of the apparatus is to make the minimum of the transmission curve broader and less deep in both cases, although curve 1 follows the original curve reasonably well except for the depth of the minimum.

The resolution width on a time of flight axis is constant as this is the directly measured quantity. The resolution at a particular energy, however, increases with decreasing energy so that for thin cadmium, although the resolution corresponded to an "on" time of $100 \mu \mathrm{sec}$. at 3 meters, the transmission minimum was much more clearly resolved.

The effect of the resolution on an absorption edge was also studied in essentially the same way, in order to examine the effect of resolution on the thick cadmium measurements. The original curve in this case was obtained by fitting two exponential functions (see curve 0, Fig. 19). - These two functions, $A\left(2-e^{-x t}\right), B e^{-x t}$, were joined at $T=0.5$ and the coefficients so adjusted that the curve corresponded to the observed transmission for thick cadmium. The derived curves are numbered to correspond to the resolution functions indicated below. Curve 1 which is indistinguishable from the original curve, was obtained for an "on" time equal to the inverse of the decay constant of the exponentials. Curves 2 and 3 were obtained for "on" times of twice and three times this amount. All the curves intersect 


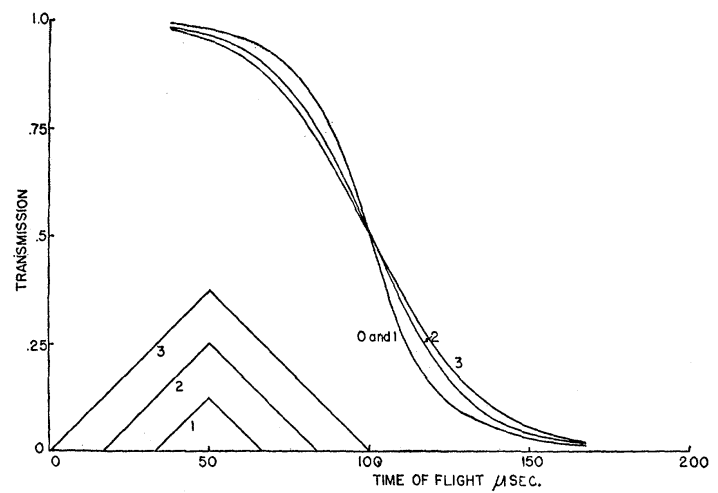

FIG. 19. Resolution of double exponential function simulating an "absorption edge." Curves 1, 2 and 3 give the effect of the resolution of the apparatus for the distribution functions indicated.

for a transmission value of one-half so that this point is given correctly regardless of the resolution used. This will, in general, be true only if the original transmission curve is symmetrical about this point. The general shape of the transmission curve is not greatly altered with any of the resolving powers illustrated. The thick cadmium measurement was made with the resolution illustrated by curve 2 . The effect of the resolution on a sharp edge was also investigated. This, of course, shows considerable effect of the resolution but the result was a much sharper break than that observed for thick cadmium.

It was also found that the resolution of the apparatus had no effect on the shape of a single exponential function, i.e., if $M(t)$ is an exponential, the derived function is also an exponential function with the same decay constant. Consequently the delayed emission results do not need to be corrected for resolution.

When the time of flight is such that there is an appreciable contribution to the beam from the thermal distribution the effect of delayed emis-. sion must be considered. The resolving power will be decreased since the neutron pulse will be broader, and the simple resolution function $f(s, t)$ will no longer apply. Furthermore, the energy to be associated with a given time of flight setting will be changed if the delayed neutrons make an important contribution. The actual importance of the delayed neutrons depends on two considerations: (1) the relative number of cascading neutrons to thermal neutrons in the energy or time of flight interval to which the apparatus is sensitive, and (2) the amount of distortion of the delayed emission tail of the pulse due to the variation in the number-energy distribution of thermal neutrons, i.e., neutrons emitted long after the pulse must have a shorter time of flight or higher energy to arrive at the detector at the same time as neutrons emitted soon after the pulse. Above an energy $E=k T=0.025 \mathrm{ev}$, this will decrease the effect of the tail as the number of neutrons decreases with increasing energy. The amount of this decrease depends on the particular energy setting.

The variation in the number-energy relationship also causes a distortion of the pulse itself, giving more weight (in a region above $E=k T$ ) to the neutrons emitted in the early part of the pulse because of the greater number of neutrons of lower energy or longer time of flight in the distribution. This distortion also affects the energy to be associated with a given setting of the apparatus. The amount of the distortion correction depends on the source-detector distance since this distance determines the spread in energy which corresponds to a given spread in time or resolution width.

The amount of distortion of the pulse and its tail can be computed with the aid of the numberenergy relationships as given by Bethe. ${ }^{7}$ These are expressed as $N(E) d E$, but to apply to this problem they must be expressed not in terms of the number as a function of energy in an energy interval, but in terms of time of flight in a time of flight interval. When they are so expressed, $Q$, the rate of neutron production taken as unity, and the values of the constants introduced, Bethe's Eq. (440) for the number of cascading neutrons becomes

$$
N(t) d t=1.8 \times 10^{-8} / d^{-1} d t,
$$

and Eq. (461) for the thermal neutrons becomes

$$
\begin{aligned}
N^{\prime}(t) d t=4.05 & \times 10^{4} d^{3} t^{-4} \\
& \times \exp \left(-2.01 \times 10^{5} d^{2} / t^{-2}\right) d t,
\end{aligned}
$$

where $t$ is the time of flight in $\mu$ sec. from source to detector and $d$ is the source detector distance in meters. It is not correct to use these expressions

${ }^{7}$ H. A. Bethe, Rev. Mod. Phys. 9, 69 (1937). See Eqs (440) and (461). 
as they stand, in order to compare the number of thermal and cascading neutrons since equilibrium of thermal neutrons with the source was assumed in the derivation of $N^{\prime}(t)$ above, and this condition is not satisfied in the problem under consideration. The cascading neutrons probably come into equilibrium with the source in a very short time (order of $10 \mu \mathrm{sec}$.) but because of the long mean life (approximately $200 \mu \mathrm{sec}$.) it takes much longer for the equilibrium number of thermal neutrons to be established. For any time of the order of the mean life the number of thermal neutrons in the paraffin increases during the burst. As the duration of the burst used in the experiments was always short compared to the time necessary to establish this equilibrium, this effect was important. If the time of slowing down to thermal energies may be neglected (10) should be multiplied by a time factor $\left(1-e^{-t^{\prime} / \tau}\right)$ where $t^{\prime}$ is measured from the start of the burst and $\tau$ is the mean life of thermal neutrons in the paraffin. This time factor also causes an additional distortion of the pulse giving more weight to the end of the pulse and therefore higher energy neutrons. At energies greater than $k T$ it is in the opposite direction to the distribution distortion.

To illustrate the effect of these corrections on the data a sample calculation was made of the effect of distortion of the pulse shape and of delayed emission. Simplified conditions of sourcedetector distance of 3 meters, source "on" time of $100 \mu \mathrm{sec}$. , and an infinitesimal detector "on" time were assumed. The relative number of neutrons from various parts of the pulse and its tail which would be recorded at the detector are given in the last five columns of Table I for time of flight settings from the center of the pulse corresponding to $0.2,0.15$, and $0.10 \mathrm{ev}$. In the second column the ratio of the number of thermal neutrons at the pulse center to the number of cascading neutrons is given. It may be seen on the basis of these assumptions that the thermal distribution is not important at an energy of $0.2 \mathrm{ev}$ and while there will be some distortion of the pulse shape at this energy the effect of the delayed emission will be in the opposite direction and can essentially be neglected. At $0.15 \mathrm{ev}$ the effect of pulse distortion is not great but the effect of the tail of the pulse begins to be appreciable and the resolution is not as good. The thermal neutrons, however, still make only about half the contribution to the number of recorded neutrons so the effect on the data is not large. At $0.10 \mathrm{ev}$ the time distortion begins to be more important than the distribution distortion and the effect of the tail is more important. Furthermore, the beam is now predominantly thermal neutrons so the energy to associate with the time setting computed from the time of flight from the center of the pulse is too low by 10 percent or more. The decrease in resolving power at this and all lower energies, begins to have an appreciable effect on the data. In this region, however, the time of flight is longer (686 $\mu \mathrm{sec}$. for 3 meters) and as the energy decreases the effect of the increasing spread in the effective "on" time becomes less important because of the increasing time of flight. For energies less than $k T$ the distortions will be markedly different, but as the data do not indicate any rapid variation in the transmission curves in this region the resolution and distortion corrections were neglected as small in magnitude and importance.

The effect of the chemical bond on the slowing down process has been neglected in the above calculations. If the effect is appreciable it will probably result in a slower loss of neutron energy which makes the thermal neutrons less important in comparison to the cascading neutrons, both because of the increased number of cascading neutrons and because of the increased delay in the production of thermal neutrons. These calculations might therefore be considered as showing the maximum expected effect, but can only be considered as indicating the order of magnitude because of the lack of detailed knowledge of the slowing down process.

TABLE I. Relative numbers of neutrons from various parts of a 100- $\mu$ sec. pulse and its tail with short detector time. $N^{\prime} / N$ gives the ratio of thermal to cascading neutrons.

\begin{tabular}{|c|c|c|c|c|c|c|}
\hline \multirow[b]{2}{*}{$\begin{array}{c}\text { ENERGY } \\
\text { EV }\end{array}$} & \multirow[b]{2}{*}{$N^{\prime} / N$} & \multicolumn{5}{|c|}{ Time in $\mu$ Sec. From the Start of the Burst } \\
\hline & & 25 & $\begin{array}{c}50 \\
\text { PULSE } \\
\text { CENTER }\end{array}$ & $\begin{array}{c}100 \\
\text { PULSE } \\
\text { END }\end{array}$ & 125 & 150 \\
\hline 0.20 & 0.33 & 0.91 & 1.0 & 0.38 & 0.13 & 0.027 \\
\hline 0.15 & 1.2 & 0.71 & 1.0 & 0.81 & 0.52 & 0.18 \\
\hline 0.10 & 3.9 & 0.60 & 1.0 & 1.26 & 0.98 & 0.60 \\
\hline
\end{tabular}




\section{Discussion of Results}

The plot of the thin cadmium data (Fig. 11) clearly shows the resolution of the resonance and gives a maximum value of the cross section of $5.4 \times 10^{-21} \mathrm{~cm}^{2}$ at $0.14 \mathrm{ev}$. Below this energy the cross section decreases to about $2.3 \times 10^{-21} \mathrm{~cm}^{2}$ and then rises slowly with decreasing energy. The minimum appears to be in the neighborhood of $0.03 \mathrm{ev}$ but since the minimum is not well defined and the points are scattered, the energy of this minimum cross section cannot be stated accurately. The cadmium resonance unfortunately comes at an energy where the corrections involving the thermal group of neutrons are most uncertain. As previously indicated, the effect of delayed emission becomes noticeable somewhere below $0.15 \mathrm{ev}$ and is an appreciable effect at 0.10 ev. The shape of the low energy side of the resonance is therefore under suspicion. At still lower energy the time of flight is long enough that the effect of delayed emission and distortion of the pulse shape have a less important effect. The relative error in the energy may still be considerable. A corrected plot of the data was made which included an approximate correction for the delayed emission but not the distortion of the pulse, thus making a considerable over-correction for the effect of the thermals. The energy of the maximum was shifted only $0.02 \mathrm{ev}$ to $0.16 \mathrm{ev}$. This seems to be sufficiently small so that for the present it is best to omit corrections for the delayed emission in the thin cadmium data. Also the ratio of cascading to thermal neutrons is not well known as a function of energy in this range because of the uncertain effect of the hydrogen chemical bond. With more complete information about the slowing down process and with more accurate data such corrections will be important.

The cross section in the region of the maximum agrees surprisingly well for the data taken at 3 meters and at 1.5 meters. These data also agree well with the data taken at still shorter distances in preliminary work. This seems to preclude the possibility of gross accidental or systematic errors in the measurement since timing errors would be expected to be constant and not to vary linearly with the distance from source to detector. Also uncorrected errors due to delayed emission or pulse distortion would affect the data taken at various distances differently.
A measurement of the cadmium cross section by the time of flight method was made by FGMTW. ${ }^{3}$ They show a high point in the cross section plot at $0.10 \mathrm{ev}$. This rough agreement is fortuitous, since the position of the high point is not sufficiently well established because of the small number of observations and the low resolution in their results. At energies below the high point they find a cross section which decreases continuously with energy, in disagreement with the present results which show a minimum and then an increasing cross section. The increasing cross section has better theoretical justification, for at sufficiently low energy the $E^{-\frac{1}{2}}$ factor in the Breit-Wigner formula will predominate and predicts a $1 / v$ cross section.

A determination of the cadmium resonance was also made by Hoffman and Livingston ${ }^{8}$ who arrived at their results by measuring the cross section in boron of those neutrons which were not absorbed in cadmium. This essentially amounts to an inference of the constants of the cadmium resonance without resolving the line. They found $E_{R}=0.18 \mathrm{ev}$ and $\Gamma=0.15 \mathrm{ev}$ and in view of this very indirect method, their results are in sufficient agreement with the present results.

In order to determine the width of the resonance, an attempt was made to fit the data with a dispersion curve. ${ }^{7} \mathrm{~A}$ point-by-point fit of the data to a dispersion curve was not feasible because of the complexity of the effect of resolution on the shape in the intermediate and thermal energy region, and because of the statistical inaccuracy of the data. A simple criterion, however, is to compare the ratio of the cross section at resonance with the cross section at some other energy, i.e., to solve Eq. (7) for $\Gamma^{2}$ and evaluate it by introducing the measured values of the cross sections.

$$
\Gamma^{2}=\frac{4\left(E-E_{R}\right)^{2}}{\left(\sigma_{0} / \sigma\right)\left(E_{R} / E\right)^{\frac{2}{2}}-1} .
$$

The ratio of the cross section at resonance to the cross section at the low energy minimum gives $\Gamma(\mathrm{Cd})=0.12 \mathrm{ev}$. Insufficient resolution would make the measured cross section at resonance lower than it actually is, but would have less effect on the minimum as it is much broader on a

${ }^{8} \mathrm{~J}$. G. Hoffman and M. S. Livingston, Phys. Rev. 52, 1228 (1937). 
time of flight axis. The observed ratio is therefore equal to or smaller than the true value and the value of $\Gamma$ computed from it is an upper limit.

A check on this value of the width can be made by considering the ratios of the cross section at resonance to the cross sections at higher energies. With the thick cadmium measurements, the ratio of the cross section at resonance to the cross sections at $0.26,0.36,0.52$ and $0.80 \mathrm{ev}$ gives values of $\Gamma$ of $0.12,0.17,0.23$ and $0.31 \mathrm{ev}$, respectively. Whether this variation of $\Gamma$ computed on the basis of a single resonance is real or whether it is entirely due to an effect of resolution has not been determined. Since the resolution correction to the thick cadmium data is about equal to the probable error in the measurements, these data could not give a conclusive answer. The resolving power of the apparatus could give rise to a discrepancy in this direction, so that the smaller value of $\Gamma$ which agrees with the result from thin cadmium is probably the best.

From a knowledge of the absolute cross section at resonance and the effective width, one can obtain the neutron width $\Gamma_{N}$ from Eq. (8). It seems likely that the absorption in cadmium is by one of the odd isotopes whose abundances are 12.3 and 13 percent. If one assumes 13 percent abundance of the effective isotope, the cross section at resonance becomes $\sigma_{R}{ }^{\prime}=4.2 \times 10^{-20}$ $\mathrm{cm}^{2}$. Since $i=\frac{1}{2}$ for the odd isotopes, $\Gamma_{N}{ }^{\prime}=3 \times 10^{-4}$, or $10^{-3} \mathrm{ev}$, depending upon the angular momentum of the resulting nucleus.

The data for thick cadmium are not subject to the uncertain corrections which must be considered for thin cadmium, since the data are taken at a higher energy where the effect of the thermal neutrons can be largely neglected. Certainly, the effect of delayed emission does not require any appreciable correction above $0.15 \mathrm{ev}$. These data fit the sample resolution curve given in the previous section very well, hence a good idea of the amount of the resolution correction can be obtained (compare curves 0 and 2, Fig. 19). The shape of the curve is not seriously altered by this effect, and probably at no point is the transmission value altered by more than 0.08 . In general, the resolution correction is of the order of magnitude of the probable error in the measurement of the transmission value.

What has been referred to as an absorption edge in cadmium is seen to be poorly defined since there is no energy where one can say that the transmission decreases rapidly. It is probably best merely to say that for the thickness used $0.45 \mathrm{~g} / \mathrm{cm}^{2}$ ), the energy for a transmission of onehalf is $0.47 \mathrm{ev}$, and that below $0.2 \mathrm{ev}$ the transmission is negligible ( 3 percent or less).

The data for indium and rhodium are also free from the uncertainties introduced by the thermal distribution since the interesting parts of the curves are in the region of higher energy. On the other hand, the shapes of the curves are definitely affected by the resolution of the apparatus. Although the smallest "on" times were used for these measurements, the energy resolution is much poorer in this region than in the region of the cadmium resonance. When one compares the data with the curves shown in Fig. 17 it is evident that the absorption line is more poorly resolved than in the hypothetical case. Although the "on" times, actually used, correspond to case 1 the resolution realized corresponds more nearly to case 2 . The thickness of the absorbers used was such that the transmission at resonance was almost certainly negligible, while the data show a transmission at resonance probably over onehalf. This indicates that the width of the line is somewhat less than was chosen for sample plots to illustrate the resolution where the smallest value of $\Gamma$ considered was $0.2 \mathrm{ev}$. Since the corrections due to the low resolution were rather large and the data of an exploratory nature, it did not seem reasonable to try to make any resolution corrections. It appears that the width is probably less than $0.2 \mathrm{ev}$, and that the energy of the minimum in the transmission is about 1.0 ev. The observed shape of the resonance, however, is not entirely due to the resolving power of the apparatus since the resolution used was such that a very sharp resonance could at most affect four adjacent points. The breadth of the absorption lines observed for rhodium and indium is, therefore, not negligible in comparison with the resolution width, even though the correction for resolution is large. No information about the cross section can be inferred from the data since absolute calibration of the transmission was not made.

The energies of the indium and rhodium resonances have been determined by the boron 
method. Goldsmith and Rasetti ${ }^{9}$ found values of $1.16 \mathrm{ev}$ and $1.38 \mathrm{ev}$ for rhodium and indium, respectively. Later measurements by Hornbostel, Goldsmith, and Manley ${ }^{10}$ on the self- and mutual absorption of the resonance neutrons in rhodium and indium give values for $\Gamma$ of 0.13 and $0.07 \mathrm{ev}$ for rhodium and indium, respectively, and the difference in the energies of the resonances as $0.15 \mathrm{ev}$. While it is difficult to see how the time of flight measurements could be in error by more than $0.2 \mathrm{ev}$, in view of the uncertainties in both methods the results are in good agreement. The widths from absorption measurements are well within the upper limit set by these results, and the difference in energy of $0.15 \mathrm{ev}$ is within the experimental error of this work.

The time of flight measurements give a direct determination of the resonance energy and the approximate agreement with the results of the boron method constitute a check on the $1 / v$ absorption in boron. A direct measurement of the boron absorption was made by Fertel $e t$ al. with results inconsistent with the $1 / v$ law. The most serious disagreement is at low energy where they found much too low a cross section. This disagreement is in the same energy region and in the same direction as their disagreement with the results of this work on cadmium, so that one is tempted to suspect some systematic error in their results at low energy. There were some experimental difficulties, and the authors state their regret that they were unable to pursue their investigations further. In view of the many good reasons for expecting the $1 / v$ law to hold, in addition to the check in this work, their results cannot be considered as a basis for serious doubt of the $1 / v$ absorption in boron, although it would be desirable to repeat the boron absorption experiment.

The study of the delayed emission was undertaken primarily to ascertain the effect upon the time of flight measurements and the experimental conditions were the same except for the detector position. It also provides some direct evidence of the existence of thermal neutrons for some considerable period after the burst although

\footnotetext{
${ }^{9} \mathrm{H}$. Goldsmith and F. Rasetti, Phys. Rev. 50, 328 (1936).

${ }_{10}$ Hornbostel, Goldsmith and Manley, Phys. Rev. 58, 18
}

the geometry of the experiment was not well suited to an accurate determination of the mean life. It was found that the effective mean life of thermal neutrons coming out of the block is $170 \mu \mathrm{sec}$. This, however, is somewhat shorter than the true mean life because of diffusion of neutrons from the inside of the block to the surface where they escape.

The effect of the diffusion can be estimated,* if one assumes as initial conditions the stationary neutron distribution due to a uniform production of thermal neutrons in an infinite slab of paraffin of thickness $2 a$. Under these conditions the neutron current at the surface of the slab is

$$
j=\frac{2 q}{a} \sum_{n \text { odd }} \frac{\exp \left[-\left(1+n^{2} \gamma^{2}\right) t / \tau\right]}{1+n^{2} \gamma^{2}} .
$$

$q$ is the number of thermal neutrons produced per $\mathrm{cm}$ per sec., $\tau$ is the true mean life of neutrons in the paraffin and $\gamma=\pi \lambda / 2 a$ where $\lambda$ is the diffusion length, approximately $2 \mathrm{~cm}$. For the source used $a$ is $10 \mathrm{~cm}$. The current $j$ was plotted logarithmically against $t / \tau$ and the best straight line approximation to this function was plotted in the time interval in which observations were taken. The slope of this line indicated that the observed decay or effective mean life, if the above conditions had been fulfilled, would have been 20 percent less than the true mean life in the paraffin. This gives a mean life of $210 \mu$ sec., but the geometrical correction in the actual conditions used (Fig. 3) is probably larger than this, thus indicating a still longer true mean life. The effect of the lack of homogeneous sources would be in the opposite direction. These results therefore cannot be used accurately to determine the true mean life. A determination of the mean life of neutrons in water was made by Frisch, v. Halban and Koch ${ }^{11}$ who arrived at the result $270 \mu \mathrm{sec}$. from a measurement of the capture cross section. Reduced to paraffin this would be $230 \mu \mathrm{sec}$. in agreement with the corrected value above.

The writers are grateful to H. A. Bethe and G. Placzek for numerous stimulating discussions during the course of these experiments.

* The writers are indebted to G. Placzek for this calculation.

${ }^{11}$ Frisch, v. Halban and Koch, Kgl. Danske Videns. Selskab. Math.-fys. Medd. 15, 10 (1937). 